\title{
Current and field driven domain wall motion under influence of the Dzyaloshinsky-Moriya interaction
}

\author{
R. Wieser \\ International Center of Quantum Materials, Peking University, \\ No. 209 Chengfu Road, Haidian District Beijing, 100871, China
}

(Dated: August 27, 2015)

\begin{abstract}
A complete analytical description of the dynamics of current and field driven transverse domain walls under the influence of the Dzyaloshinsky-Moriya interaction using the $\phi-q$ model will be given. Five different scenarios will be observed where the Dzyaloshinsky-Moriya vector is either parallel or perpendicular to the easy axis anisotropy of the system and a direct reversal respectively precessional motion will be assumed.

PACS numbers: 75.78.-n, 75.60.Ch, 75.10.Hk
\end{abstract}

\section{INTRODUCTION}

Due to the usability for data storage 1, 2], information transport [3] and as logic elements [4, [5] magnetic wires and spin chains became more and more important during the last years. An important role in all these applications play the motion of domain walls which underlies some criteria: it shall be fast, controllable and stable.

While the domain wall motion of a normal transverse domain wall is well understood the influence of the Dzyaloshinsky-Moriya interaction is mostly tuned out. However, the Dzyaloshinsky-Moriya interaction is induced by the spin-orbit interaction and appears if the symmetry of the system is broken. This is the case at surfaces. Now, nearly all magnetic nanowires and spin chains are deposit on a surface. Therefore, the influence of a Dzyaloshinsky-Moriya interaction on the domain wall motion should not be ignored.

A first overview of the influence of the DzyaloshinskyMoriya interaction on the motion a transverse domain wall has been given in the pioneering works of Tretiakov and Abanov [ [], and Thiaville et al. [7]. However, these publications don't cover all possible scenarios. While [6] describes the influence of a Dzyaloshinsky-Moria vector parallel to the easy axis directions in the case of a current driven domain wall, [7] describes the influence of a Dzyaloshinsky-Moria vector perpendicular to the easy axis driven by an external field. Furthermore, in 7] the description is restricted to the direct reversal scenario during the domain wall motion. The possibility of a precession during the domain wall motion has been ignored. Therefore, we can say so far there is no publication which describes the field and current driven domain wall motion under the influence of the Dzyaloshinsky-Moriya interaction, which covers all possible scenarios. This leak of information shall be closed with this publication. Furthermore, the description given by Tretiakov and Abanov is quite complex. This publication presents a simple way which is easy to understand and which offers a deeper understanding.

The next section introduces the $q-\phi$ model which has been used to investigate the domain wall motion of a transverse domain wall under the influence of the Dzyaloshinsky-Moriya interaction.

\section{II. $q-\phi$ MODEL}

A starting point for the analytical description of any kind of domain wall structures and their dynamics is the Micromagnetic continuum approximation of the classical Heisenberg model. The magnetic properties of a 1D or quasi-1D domain wall with influence coming from the Dzyaloshinsky-Moriya interaction are well described by the following Heisenberg Hamiltonian:

$$
\begin{aligned}
\mathcal{H} & =-J \sum_{\langle i j\rangle} \vec{S}_{i} \cdot \vec{S}_{j}-\vec{D}_{\mathrm{DM}} \cdot \sum_{\langle i j\rangle} \vec{S}_{i} \times \vec{S}_{j}-\mu_{S} B_{z} \sum_{i} S_{i}^{z} \\
& +D_{h} \sum_{i}\left(S_{i}^{y}\right)^{2}-D_{e} \sum_{i}\left(S_{i}^{z}\right)^{2},
\end{aligned}
$$

where $\vec{S}_{i}=\vec{\mu} / \mu_{S}$ are 3D magnetic moments of unit length.

The first term of this Hamiltonian describes the ferromagnetic exchange coupling between nearest neighbors with the coupling constant $J>0$. The second term describes the asymmetric exchange (Dzyaloshinsky-Moriya interaction) between nearest neighbors coming from the spin-orbit coupling. Depending on the symmetry of the system the Dzyaloshinsky-Moriya vector $\vec{D}_{\mathrm{DM}}$ is oriented perpendicular or parallel to the bonding axis of the neighboring spins $\vec{S}_{i}$ and $\vec{S}_{j}[8]$. The third sum describes the influence of an external magnetic field $B_{z}$ in $z$ direction and the last two terms represent uniaxial anisotropies with a hard axis oriented in $\pm y$ direction and an easy axis oriented in $\pm x$ direction. Within this manuscript we will assume orientations of the Dzyaloshinsky-Moriya vector $\vec{D}_{\text {DM }}$ parallel respectively perpendicular to the easy axis anisotropy. The precise orientation will be announced at the beginning of every subsection.

The Hamiltonian Eq. (11) can be used to calculate the dynamics of domain wall numerical [9 11]. However, a discrete structure is not convenient for an analytical description. In these cases it is comfortable to use the mi- 
cromagnetic continuum description. The corresponding energy:

$$
E=\int_{-\infty}^{+\infty} \mathcal{E}(\theta, \phi) \mathrm{d} z
$$

can be got from $\mathcal{H}$ by performing a Taylor expansion up to the first order [12]. Furthermore, it makes sense to use spherical instead of the cartesian coordinates: $S_{x}=\sin \theta \cos \phi, S_{y}=\sin \theta \sin \phi$, and $S_{z}=\cos \theta$. The detailed energy densities $\mathcal{E}$ corresponding to the different scenarios will be given in Sec. III and IV.

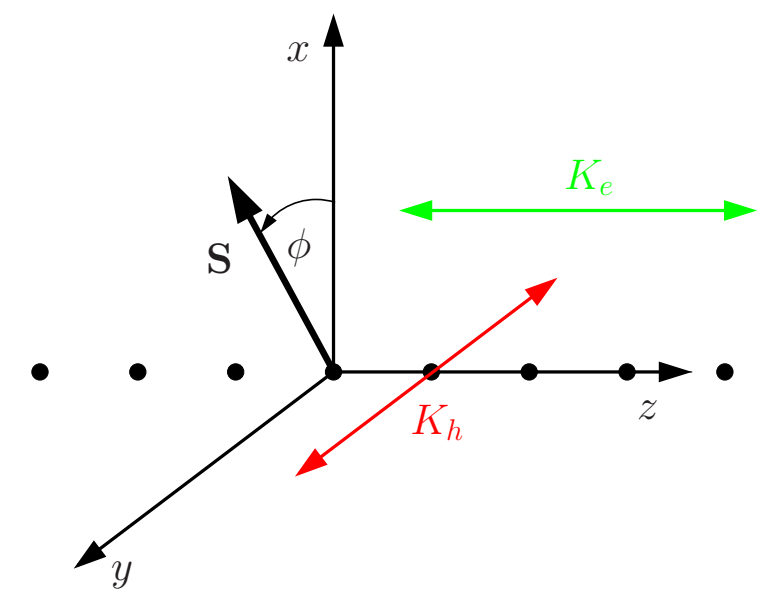

FIG. 1: Underlying coordinate system: $K_{e}$ and $K_{h}$ are the uniaxial easy respectively hard axis anisotropy of the system. $\phi$ is the angle between the magnetic moment $\mathbf{S}$ in the center of the domain wall and the $x$ axis; $\theta$ with respect to the $z$ axis (not show). The dots symbolizes the next lattice sites of the 1D or quasi-1D spin system.

Our goal is to give a complete description of the dynamics of a $180^{\circ}$ transverse domain wall in a $1 \mathrm{D}$ or quasi$1 \mathrm{D}$ system with long axis in $z$ direction. Due to this fact we assume for all scenarios that the easy axis anisotropy is oriented in $\pm z$ direction to take into account the contributions from crystalline anisotropy as well as dipoledipole interaction [9]. Furthermore, if $D_{h} \neq 0$ (additional hard axis anisotropy) the hard axis is oriented in $\pm y$ direction (see Fig. 11). Under these assumptions in all cases the magnetic moments in the domains are oriented in $\pm z$ direction and the orientation of the magnetic moment in the center of the domain wall depends on $D_{h}$ : if $D_{h}>0$ the magnetic moment will be oriented in one of the $x$ directions $( \pm x, \phi=0$ or $\phi=\pi)$, if $D_{h}=0$ the symmetry in the $x y$-plane is not broken and the magnetic moment can be oriented in any of the possible directions in the $x y$-plane $(0 \leq \phi \leq 2 \pi)$. So far we haven't take into account the Dzyaloshinsky-Moriya interaction. In the following we assume that the Dzyaloshinsky-Moriya interaction is small with respect to the other energy contributions (exchange and anisotropy): $\left|\vec{D}_{\mathrm{DM}}\right|=D_{\mathrm{DM}} \ll J$,
$D_{\mathrm{DM}} \ll D_{e}$, and $D_{\mathrm{DM}} \ll D_{h}$. The assumption of a small Dzyaloshinsky-Moriya interaction is necessary to find domain wall solutions instead of spin spirals.

Within the micromagnetic continuum description it is easy to verify that with the assumptions made before the static solution $\left(B_{z}=0\right)$ of a $180^{\circ}$ transverse domain wall is given by 12 14]:

$$
\theta(z, t)=2 \arctan \left(e^{-\frac{z-q(t)}{\Delta}}\right)
$$

and

$$
S_{z}=\cos \theta= \pm \tanh \left(\frac{z-q(t)}{\Delta}\right) .
$$

This profile is the general solution of a $1 \mathrm{D}$ or quasi1D $180^{\circ}$ domain wall and independent from the explicit form of the Hamiltonian given by Eq. (1), as long as the Dzyaloshinsky-Moriya interaction is small: $\theta$ is the angle with respect to the $z$ axis, $\Delta$ the domain wall width, which depends on $\mathcal{H}$, and $q(t)$ is the center of the domain wall. The center of the domain wall itself is characterized by $S_{z}=\cos \theta=0$ which means $\theta=\pi / 2$. Additional, we find for the variation $\delta \mathcal{E} / \delta \theta$ of the microscopic energy density $\mathcal{E}$ under the condition to be in the center of the domain wall $(\theta=\pi / 2)$ :

$$
\left.\frac{\delta \mathcal{E}}{\delta \theta}\right|_{\theta=\pi / 2}=M_{S} B_{z}
$$

A detailed description is not necessary at this point and will be given later in Sec. III and IV As before in Eq. (1) $B_{z}$ is the external field. $M_{S}=\mu_{S} / a^{3}$ is the saturation magnetization, and $a$ is the lattice constant. Additional we can deduce the following conditions from the domain wall profile:

$$
\frac{\mathrm{d} \theta}{\mathrm{d} z}=-\frac{\sin \theta}{\Delta}
$$

and

$$
v=\frac{\mathrm{d} q}{\mathrm{~d} t}=\frac{\Delta}{\sin \theta} \frac{\mathrm{d} \theta}{\mathrm{d} t},
$$

where $v$ is the velocity of the domain wall.

The underlying equation of motion is the Gilbert equation with additional spin torque terms (adiabatic and non-adiabatic) describing the influence of an electric current $u_{z} \propto j$, with $j$ the current density [10, 11]:

$$
\begin{aligned}
\frac{\mathrm{d} \vec{S}}{\mathrm{~d} t} & =\gamma \vec{S} \times \mathbf{H}_{\mathrm{eff}}-\alpha \vec{S} \times \frac{\mathrm{d} \vec{S}}{\mathrm{~d} t} \\
& -u_{z} \frac{\mathrm{d} \vec{S}}{\mathrm{~d} z}+\beta u_{z} \vec{S} \times \frac{\mathrm{d} \vec{S}}{\mathrm{~d} z} .
\end{aligned}
$$

The first term of this equation describe the precessional motion of the magnetic moment $\vec{S}$ in the effective field $\mathbf{H}_{\text {eff }}=-\left(1 / \mu_{S}\right)(\mathrm{d} \mathcal{H} / \mathrm{d} \overrightarrow{\mathcal{S}}) . \quad \gamma$ is the gyromagnetic ratio. The second term is the so called Gilbert damping with 
damping constant $\alpha$ [15]. The third and fourth terms are the adiabatic and non-adiabatic spin torque terms describing the influence of an polarized electric current. The adiabatic spin torque term appears due to the momentum conservation during the interaction between the localized magnetic moments $\vec{S}$ and the spins of the electrons. The non-adiabatic spin torque term takes into account the scattering processes of the electrons. $\beta$ is the so called non-adiabaticity constant. For a detailed description see e.g. [16, 17]. The corresponding micromagnetic equations in spherical coordinates are:

$$
\frac{\mathrm{d} \theta}{\mathrm{d} t}=\frac{\gamma}{M_{S} \sin \theta} \frac{\delta \mathcal{E}}{\delta \phi}-\alpha \sin \theta \frac{\mathrm{d} \phi}{\mathrm{d} t}-u_{z} \frac{\mathrm{d} \theta}{\mathrm{d} z}-\beta u_{z} \sin \theta \frac{\mathrm{d} \phi}{\mathrm{d} z},
$$

and

$$
\frac{\mathrm{d} \phi}{\mathrm{d} t}=-\frac{\gamma}{M_{S} \sin \theta} \frac{\delta \mathcal{E}}{\delta \theta}+\frac{\alpha}{\sin \theta} \frac{\mathrm{d} \theta}{\mathrm{d} t}-u_{z} \frac{\mathrm{d} \phi}{\mathrm{d} z}+\frac{\beta u_{z}}{\sin \theta} \frac{\mathrm{d} \theta}{\mathrm{d} z} .
$$

$\delta \mathcal{E} / \delta \theta$ respectively $\delta \mathcal{E} / \delta \phi$ are the variations of the energy density with respect to $\theta$ respectively $\phi$.

Inserting the conditions Eq. (5)-(7), together with the assumption to be in the center of the domain wall: $\theta=\pi / 2$, lead to the following equations which are the starting point of our considerations:

$$
\frac{v}{\Delta}=\frac{\gamma}{M_{S}} \frac{\delta \mathcal{E}}{\delta \phi}-\alpha \frac{\mathrm{d} \phi}{\mathrm{d} t}+\frac{u_{z}}{\Delta}-\beta u_{z} \frac{\mathrm{d} \phi}{\mathrm{d} z},
$$

and

$$
\frac{\mathrm{d} \phi}{\mathrm{d} t}=-\gamma B_{z}+\frac{\alpha v}{\Delta}-u_{z} \frac{\mathrm{d} \phi}{\mathrm{d} z}-\frac{\beta u_{z}}{\Delta}
$$

The first equation (11) describes the velocity $v$ and the second equation (12) the precession of the domain wall.

These equations are simple to solve and depend only on $q($ or $v=\mathrm{d} q / \mathrm{d} t)$ and $\phi$. In the next sections the explicit form of $\phi(z, t)$ under the assumptions of a DzyaloshinskyMoriya interaction with a vector $\vec{D}_{\mathrm{DM}}$ parallel and perpendicular to the easy axis anisotropy shall be characterized and the corresponding equations (11) and (12) solved.

A detailed outline of the following sections can be found in following table: the first and second column give the section and sketch (Fig.) of one scenario and the last four column the corresponding informations about the used assumptions (orientation of $\vec{D}_{\mathrm{DM}}, D_{e}$ and $D_{h}$ as well as the reversal mechanism where direct means $\mathrm{d} \phi / \mathrm{d} t=0$ and precession $\mathrm{d} \phi / \mathrm{d} t \neq 0)$.

This table can be seen as a road map of this publication. The calculations themselves (dealing with main equations Eq. (11) and (12)) are simple and follow always the same procedure: After writing down the energy
TABLE I:

\begin{tabular}{|c|c|c|c|c|c|}
\hline Sec. & Fig. & $D_{e}$ & $D_{h}$ & $\vec{D}_{\mathrm{DM}}$ & reversal \\
\hline III A & 2 & $\pm z$ & $\pm y$ & $+z$ & direct \\
\hline \hline III B & 3 & $\pm z$ & no anisotropy & $+z$ & precession \\
\hline \hline IV A & 4 & $\pm z$ & $\pm y$ & $+y$ & direct \\
\hline \hline IV B & 5 & $\pm z$ & $\pm y$ & $+x$ & direct \\
\hline \hline IV C & 6 & $\pm z$ & no anisotropy & $+y$ & precession \\
\hline \hline
\end{tabular}

density $\mathcal{E}$, corresponding to $\mathcal{H}$ [Eq. (10)] and the made assumptions (see table I) , the variation $\delta \mathcal{E} /\left.\delta \phi\right|_{\theta=\pi / 2}=0$ has to be calculated. Inserting the result in the main equations Eq. (11) and (12) together with an assumption about the angle $\phi$ lead to the final equations describing the domain wall motion. Independent of the chosen assumptions $\delta \mathcal{E} / \delta \theta=0$ lead to the domain wall profile Eq. (4) together with the domain wall width $\Delta$.

\section{DM VECTOR PARALLEL TO THE EASY AXIS ANISOTROPY DIRECTIONS}

In the following we assume a spin chain with a transverse domain wall with an easy axis anisotropy in $\pm z$ direction. We will stay with this assumption till the end of this publication. Furthermore, in this section we assume that the Dzyaloshinsky-Moriya vector is parallel to the easy axis direction: $\vec{D}_{\mathrm{DM}}=D_{\mathrm{DM}} \vec{e}_{z}$. Then in the following two subsections the two possible scenarios $D_{h} \neq 0$ and $D_{h}=0$ will be discussed. In the first scenario the hard axis anisotropy $D_{h}$ leads to a symmetry break and a direct reversal of the magnetic moments during the motion. In the second scenario (no hard axis anisotropy, $\left.D_{h}=0\right)$ the system shows a rotational symmetry around the long axis ( $z$ axis).

In both cases the micromagnetic energy density $\mathcal{E}$ is given by:

$$
\begin{aligned}
\mathcal{E} & =A\left[\left(\frac{\mathrm{d} \theta}{\mathrm{d} z}\right)^{2}+\sin ^{2} \theta\left(\frac{\mathrm{d} \phi}{\mathrm{d} z}\right)^{2}\right]+\mathcal{D}_{\mathrm{DM}} \sin ^{2} \theta \frac{\mathrm{d} \phi}{\mathrm{d} z} \\
& -M_{S} B_{z} \cos \theta+K_{h} \sin ^{2} \theta \sin ^{2} \phi-K_{e} \cos ^{2} \theta
\end{aligned}
$$

where $A=J / 2 a$ is the exchange stiffness, $K_{\eta}=D_{\eta} a^{3}$ $(\eta \in\{e, h\})$ the uniaxial anisotropies, $B_{z}$ the external field, $\mathcal{D}_{\mathrm{DM}}=D_{\mathrm{DM}} / a^{2}$ the micromagnetic DzyaloshinskyMoriya interaction, $M_{S}=\mu_{S} / a^{3}$ the magnetization, and $a$ the lattice constant. The difference between both scenarios is the appearance of the hard axis anisotropy: first scenario $K_{h} \neq 0$ (direct reversal), second scenario $K_{h}=0$ (precessional motion).

In the following we will continue with the micromagnetic description instead of the discrete description used in Eq. (11). 


\section{A. Direct Reversal}

In this subsection we assume a hard axis anisotropy $K_{h} \neq 0$ in $\pm y$ direction which is dominating: $K_{h} \gg$ $\mathcal{D}_{\text {DM }}$. This immediately leads to the fact that we can expect: $\mathrm{d} \phi / \mathrm{d} z=0$. In principle due to $K_{h} \gg \mathcal{D}_{\mathrm{DM}}$ the Dzyaloshinsky-Moriya interaction has no influence and we can expect in this case the well known results of domain wall motion without Dzyaloshinsky-Moriya interaction.

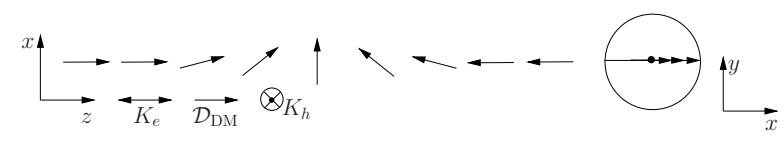

FIG. 2: Sketch of the domain wall profile of a 1D transverse domain wall with Dzyaloshinsky-Moriya interaction corresponding to the scenario described in Sec. IIIA left hand side $x-z$ plane, right hand side (circle) $x-y$ plane. The Dzyaloshinsky-Moriya vector $\mathcal{D}_{\mathrm{DM}}$ is oriented parallel to the easy axis $K_{e}$ and perpendicular to the hard axis anisotropy $K_{h}$. Due to the hard axis anisotropy $K_{h}$ we can assume a direct reversal $\mathrm{d} \phi / \mathrm{d} t=0$.

It is quite easy to verify that in this case $\delta \mathcal{E} / \delta \theta=0$ if we assume $B_{z}=0$ (static solution) for this moment leads to the well known domain wall profile (3), with the domain wall width:

$$
\Delta=\sqrt{\frac{A}{K_{e}+K_{h} \sin ^{2} \phi}} .
$$

Then, with $\delta \mathcal{E} / \delta \phi$ under the assumption to be in the center of the domain wall: $\cos \theta=0 \Rightarrow \theta=\pi / 2$ :

$$
\left.\frac{\delta \mathcal{E}}{\delta \phi}\right|_{\theta=\frac{\pi}{2}}=K_{h} \sin (2 \phi)
$$

the equations (11) and (12) become:

$$
\frac{v}{\Delta}=\frac{K_{h} \gamma}{M_{S}} \sin (2 \phi)+\frac{u_{z}}{\Delta}
$$

and

$$
0=-\gamma B_{z}+\frac{\alpha v}{\Delta}-\frac{\beta u_{z}}{\Delta} .
$$

Here, the domain wall width $\Delta$ is given by Eq. (14).

Inserting Eq. (17) in (16) leads to the condition which describes the stability of the domain wall (Walker breakdown):

$$
\phi=\frac{1}{2} \arcsin \left(\frac{M_{S} B_{z}+(\beta-\alpha) \frac{u_{z} M_{S}}{\gamma \Delta}}{\alpha K_{h}}\right) .
$$

Inserting this result in Eq. (14), gives the domain wall width $\Delta$ during the domain wall motion e.g. $B_{z} \neq 0$. Furthermore, Eq. (16) together with Eq. (18) leads to the well known velocity equation for a domain wall motion with direct reversal [9, 11, 18]:

$$
v=\frac{\gamma B_{z}}{\alpha} \Delta+\frac{\beta}{\alpha} u_{z} .
$$

The first term describes the influence of an external field and the second term the influence of the electric current. The same result can be derived also directly from Eq. (17).

As discussed before the dominating hard axis anisotropy $K_{h}$ leads to the fact that the DzyaloshinskyMoriya interaction has no influence. This can be seen also in the equation for the velocity Eq. (41) and stability equation (18). The equations for the velocity as well as the stability of the domain wall are the same as in this scenario. For comparison, the domain wall motion of a transverse domain wall without influence of a Dzyaloshinsky-Moriya interaction is described in [11, 18].

On the other hand the assumption of a dominating hard axis anisotropy $K_{h}$ is a quite hard criteria which was necessary to solve this problem analytical. A softer assumption: $K_{h}$ not dominating will lead to an influence of the Dzyaloshinsky-Moriya interaction. However, in this case a complete analytical description without approximations is impossible, e.g. Tretiakov and Abanov [6] have used a perturbation theory to obtain their result.

\section{B. Precessional motion}

While $K_{h} \neq 0$ is not exactly solvable without the assumption of a dominating hard axis anisotropy the situation changes if we assume $K_{h}=0$ (no hard axis anisotropy). As before in previous subsection we assume the Dzyaloshinsky-Moriya vector parallel to the easy axis anisotropy in $+z$ direction. Furthermore, we assume $K_{h}=0$, therefore we have a rotational symmetry around the $z$ axis and this means we cannot neglect the influence of the Dzyaloshinsky-Moriya interaction: $\mathrm{d} \phi / \mathrm{d} z \neq 0$. Furthermore, we have to expect that the magnetic moments and therefore the domain wall precess during the motion: $\mathrm{d} \phi / \mathrm{d} t \neq 0$.

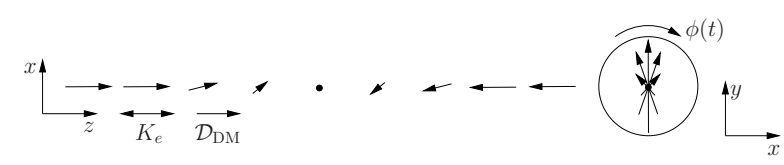

FIG. 3: Sketch of the domain wall profile of a 1D transverse domain wall with Dzyaloshinsky-Moriya interaction corresponding to the scenario described in Sec. IIIB left hand side $x-z$ plane, right hand side (circle) $x-y$ plane. The Dzyaloshinsky-Moriya vector $\mathcal{D}_{\mathrm{DM}}$ is oriented parallel to the easy axis anisotropy $K_{e}$. Due to the Dzyaloshinsky-Moriya interaction and the absence of the hard axis anisotropy $K_{h}=0$ the domain wall profile shows a twist and we have to assume a precessional motion $\mathrm{d} \phi / \mathrm{d} t \neq 0$. 
In this scenario $\delta \mathcal{E} / \delta \phi=0$ leads to:

$$
\frac{\delta \mathcal{E}}{\delta \phi}=-\frac{\mathrm{d}}{\mathrm{d} z}\left[\sin ^{2} \theta\left(2 A \frac{\mathrm{d} \phi}{\mathrm{d} z} \pm \mathcal{D}_{\mathrm{DM}}\right)\right]=0,
$$

which means:

$$
\frac{\mathrm{d} \phi}{\mathrm{d} z}=\mp \frac{\mathcal{D}_{\mathrm{DM}}}{2 A}
$$

The solution of this differential equation is given by:

$$
\phi(z, t)=\mp \Gamma(z-q(t))+\phi_{0},
$$

where we have set $z_{0}=q$ and $\Gamma=\mathcal{D}_{\mathrm{DM}} / 2 A$. This equation describes the linear increase / decrease of $\phi$ with $z$ due to the Dzyaloshinsky-Moriya interaction. In other words we find a twist in the domain wall profile: $\mathrm{d} \phi / \mathrm{d} z \neq 0$ (see Fig. 3).

With this knowledge it is easy to show that $\delta \mathcal{E} / \delta \theta=0$, with $B_{z}=0$, leads to the well known domain wall profile (3) together with the domain wall width:

$$
\Delta=\sqrt{\frac{A}{K_{e}-\frac{\mathcal{D}_{\mathrm{DM}}^{2}}{4 A}}}
$$

To describe the dynamics it is necessary to know $\delta \mathcal{E} / \delta \phi$ and $\mathrm{d} \phi / \mathrm{d} z: \delta \mathcal{E} / \delta \phi$ is equal to zero [see Eq. [20] ] and $\mathrm{d} \phi / \mathrm{d} z$ is described by Eq. (21). With this information we can write down our main equations Eq. (11) and (12) as:

$$
\begin{aligned}
\frac{v}{\Delta} & =-\alpha \frac{\mathrm{d} \phi}{\mathrm{d} t}+\frac{u_{z}}{\Delta} \pm \beta u_{z} \Gamma \\
\frac{\mathrm{d} \phi}{\mathrm{d} t} & =\frac{\alpha v}{\Delta}-\gamma B_{z} \pm u_{z} \Gamma-\frac{\beta u_{z}}{\Delta} .
\end{aligned}
$$

Inserting (25) in 24) we get:

$$
v=\frac{\gamma B_{z}}{\alpha+\frac{1}{\alpha}} \Delta+\frac{1+\alpha \beta}{1+\alpha^{2}} u_{z} \mp \frac{\alpha-\beta}{1+\alpha^{2}} \Gamma \Delta u_{z} .
$$

This result is identical to the velocity of a field and current driven domain wall without Dzyaloshinsky-Moriya interaction [11, 18], just with the additional term $\mp(\alpha-\beta) /\left(1+\alpha^{2}\right) \Gamma \Delta u_{z}$ describing the influence of the Dzyaloshinsky-Moriya interaction. This term increases / decreases the velocity depending on the direction of the Dzyaloshinsky-Moriya vector.

Inserting (24) in (25) leads to:

$$
\frac{\mathrm{d} \phi}{\mathrm{d} t}=\frac{\alpha-\beta}{1+\alpha^{2}} \frac{u_{z}}{\Delta}-\frac{\gamma B_{z}}{1+\alpha^{2}} \pm \frac{(1+\alpha \beta) \Gamma}{1+\alpha^{2}} u_{z} .
$$

This equation describes the precession of the transverse component of the domain wall during the motion.

At this point it should be noticed that we have assumed to be in the center of the domain wall. This means that we are in a moving frame. In the case of the domain wall velocity this is not a problem, because the velocity of the domain wall and the velocity of the moving frame are identical. However, due to the twisted shape of the domain wall not only the domain wall rotates during the motion but also our coordinate system. This rotation comes from the twisted shape but does not take into account the precessional motion of the magnetic moments. In other words Eq. (27) leads to wrong results because we are not in a stationary frame or in the rotating frame with the correct rotational frequency. In the later case we can expect $\mathrm{d} \phi / \mathrm{d} t=0$. So, if we are interested in investigating the oscillation of the domain wall we have to be in a stationary or rotating frame with the same rotational frequency as the magnetic moments. However, both is not the case. Therefore, we have to make an additional transformation to get in one of these two frames. In the following we use the stationary frame.

We know from Eq. (21) that:

$$
\frac{\mathrm{d} \phi}{\mathrm{d} z}=\mp \Gamma \quad \Leftrightarrow \quad \mathrm{d} \phi=\mp \Gamma \mathrm{d} z
$$

and therefore:

$$
\frac{\mathrm{d} \phi}{\mathrm{d} t}=\mp \Gamma \frac{\mathrm{d} z}{\mathrm{~d} t}
$$

This is the correction we have to take into account to change from the rotating to the stationary frame. $v=\mathrm{d} z / \mathrm{d} t$ is the velocity of the domain wall and at the same time the velocity of the moving frame. Adding this term to Eq. (27) and taking into account Eq. (26), the precession in the stationary frame is described by:

$$
\frac{\mathrm{d} \phi}{\mathrm{d} t}=\frac{\alpha-\beta}{1+\alpha^{2}}\left(\frac{1}{\Delta} \pm \Gamma^{2} \Delta\right) u_{z}-\frac{\gamma B_{z}}{1+\alpha^{2}}(1 \pm \alpha \Gamma \Delta) .
$$

Together with equation (26) this equation describes the precessional domain wall motion of transverse domain walls with a Dzyaloshinsky-Moriya interaction where the Dzyaloshinsky-Moriya vector is parallel to the easy axis anisotropy. This result is identical with the result given by Tretiakov and Abanov [6], however the given description here is simpler.

\section{DZYALOSHINSKY-MORIYA VECTOR PERPENDICULAR TO THE EASY AXIS ANISOTROPY DIRECTIONS}

The assumption of the previous section was that the Dzyaloshinsky-Moriya vector is parallel to the easy axis anisotropy. In principle this not the general situation. Theoretically, the Dzyaloshinsky-Moriya vector can show in any direction, depending on the symmetry of the system. To simplify the problem and due to the fact that we are interested in analytical solvable problems we restrict ourself to scenarios with the Dzyaloshinsky-Moriya vector perpendicular to the easy axis anisotropy. In the following, we investigate three scenarios: (1) the Dzyaloshinsky-Moriya vector has an orientation perpendicular to the easy axis anisotropy $K_{e}$ and parallel to 
the hard axis anisotropy $K_{h},(2)$ the DzyaloshinskyMoriya vector is perpendicular to the easy as well hard axis anisotropy $K_{h}$, and (3) there is just an easy axis anisotropy $\left(K_{h}=0\right)$. In all these scenarios we assume that the spin chain has an alignment along the $z$ axis, the easy axis is oriented in $\pm z$ direction (transverse domain wall) and the Dzyaloshinsky-Moriya vector is oriented in $y$ direction. To distinguish the three scenarios we change the strength (1),(2) $K_{h} \neq 0,(3) K_{h}=0$ and orientation of the hard axis anisotropy $K_{h}$ : (1) the hard axis anisotropy $K_{h}$ is oriented in $\pm y$ direction, (2) in $\pm x$ direction.

The micromagnetic energy density $\mathcal{E}$ in the case of a Dzyaloshinsky-Moriya vector in $y$ direction parallel to the hard axis anisotropy $K_{h}$ is given by:

$$
\begin{aligned}
\mathcal{E} & =A\left[\left(\frac{\mathrm{d} \theta}{\mathrm{d} z}\right)^{2}+\sin ^{2} \theta\left(\frac{\mathrm{d} \phi}{\mathrm{d} z}\right)^{2}\right]+\mathcal{D}_{\mathrm{DM}} \cos \phi \frac{\mathrm{d} \theta}{\mathrm{d} z} \\
& -M_{S} B_{z} \cos \theta+K_{h} \sin ^{2} \theta \sin ^{2} \phi-K_{e} \cos ^{2} \theta
\end{aligned}
$$

For a hard axis anisotropy $K_{h}$ in $\pm x$ direction we have to replace the term $K_{h} \sin ^{2} \theta \sin ^{2} \phi$ by $K_{h} \sin ^{2} \theta \cos ^{2} \phi$.

\section{A. Direct Reversal: Dzyaloshinsky-Moriya vector parallel to $K_{h}$}

As before in the first scenario, we assume the appearance of both uniaxial anisotropies $K_{e}$ and $K_{h}$. However, this time we assume that the Dzyaloshinsky-Moriya vector $\mathcal{D}_{\mathrm{DM}}$ shows in $+y$ direction (parallel to $K_{h}$ ) and not in $+x$ direction. In this case we don't need to assume a dominating hard axis anisotropy to solve the problem exactly.

In this scenario the variation $\delta \mathcal{E} / \delta \theta$, with $B_{z}=0$, leads to the following result:

$$
\begin{aligned}
\frac{\delta \mathcal{E}}{\delta \theta} & =2 A \frac{\mathrm{d}^{2} \theta}{\mathrm{d} z^{2}}-\mathcal{D}_{\mathrm{DM}} \sin \phi \frac{\mathrm{d} \phi}{\mathrm{d} z} \\
& +2 \sin \theta \cos \theta\left[K_{e}+K_{h} \sin ^{2} \phi-A\left(\frac{\mathrm{d} \phi}{\mathrm{d} z}\right)^{2}\right]=0
\end{aligned}
$$

With the assumption that $\phi$ is constant: $\mathrm{d} \phi / \mathrm{d} z=0$ and $\mathrm{d} \phi / \mathrm{d} t=0$ this differential equation can be solved easily and leads again to the well known domain wall profile (3) together with an domain wall width which is not influenced by the Dzyaloshinsky-Moriya interaction:

$$
\Delta=\sqrt{\frac{A}{K_{e}+K_{h} \sin ^{2} \phi}} .
$$

The second variation $\delta \mathcal{E} / \delta \phi$, under the assumption to be in the center of the domain wall: $\theta=\pi / 2$ and a constant $\phi$, leads to:

$$
\frac{\delta \mathcal{E}}{\delta \phi}=\left(2 K_{h} \cos \phi+\frac{\mathcal{D}_{\mathrm{DM}}}{\Delta}\right) \sin \phi .
$$

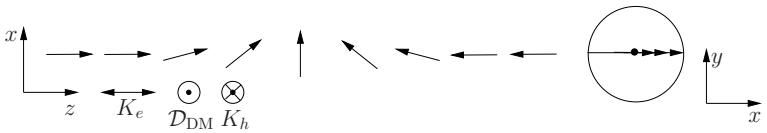

FIG. 4: Sketch of the domain wall profile of a 1D transverse domain wall with Dzyaloshinsky-Moriya interaction corresponding to the scenario described in Sec. IVA left hand side $x-z$ plane, right hand side (circle) $x-y$ plane. The Dzyaloshinsky-Moriya vector $\mathcal{D}_{\mathrm{DM}}$ is oriented perpendicular to the easy axis $K_{e}$ and parallel to the hard axis anisotropy $K_{h}$. The Dzyaloshinsky-Moriya interaction only affects the domain wall width but not the profile itself. Due to the hard axis anisotropy $K_{h}$ we can assume a direct reversal $\mathrm{d} \phi / \mathrm{d} t=0$.

Therefore, we are able to write the main equations Eq. (11) and (12) as:

$$
\begin{aligned}
\frac{v}{\Delta} & =\frac{\gamma}{M_{S}}\left(2 K_{h} \cos \phi+\frac{\mathcal{D}_{\mathrm{DM}}}{\Delta}\right) \sin \phi+\frac{u_{z}}{\Delta} \\
0 & =\frac{\alpha v}{\Delta}-\gamma B_{z}-\frac{\beta u_{z}}{\Delta} .
\end{aligned}
$$

After eliminating $v$ we get the stability condition:

$$
\sin \phi=\frac{\gamma B_{z}+(\beta-\alpha) \frac{u_{z}}{\Delta}}{\frac{\alpha \gamma}{M_{S}}\left(2 K_{h} \cos \phi+\frac{\mathcal{D}_{\mathrm{DM}}}{\Delta}\right)} .
$$

Inserting this result in (35) gives the formula for the velocity:

$$
v=\frac{\gamma B_{z}}{\alpha} \Delta+\frac{\beta}{\alpha} u_{z} .
$$

This result is identical to the velocity of a transverse domain wall with direct reversal and no DzyaloshinskyMoriya interaction [11, 18]. The only difference between both descriptions is the stability criteria (37). In the case with Dzyaloshinsky-Moriya interaction the domain walls are more stable, which means the Walker breakdown appears at higher field and current values.

\section{B. Direct Reversal: Dzyaloshinsky-Moriya vector perpendicular to $K_{h}$}

In the following we assume the same geometry as before, however with the following difference: the Dzyaloshinsky-Moriya vector is now oriented in $+x$ direction and therefore perpendicular to both anisotropies $K_{e}$ and $K_{h}$ (see Fig. 5 ).

This scenario is solvable if we assume that both anisotropies are dominating with respect to Dzyaloshinsky-Moriya interaction: $K_{h} \gg \mathcal{D}_{\mathrm{DM}}, K_{e} \gg$ $\mathcal{D}_{\mathrm{DM}}$. In this case the Dzyaloshinsky-Moriya interaction can be neglected and we find a normal transverse domain wall with the profile (3) and the domain wall width:

$$
\Delta=\sqrt{\frac{A}{K_{e}+K_{h} \sin ^{2} \phi}} .
$$




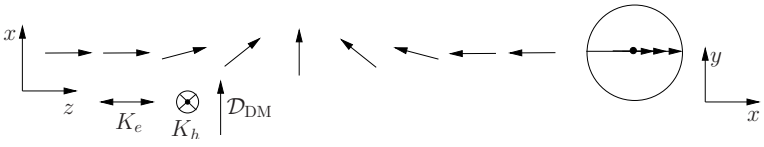

FIG. 5: Sketch of the domain wall profile of a 1D transverse domain wall with Dzyaloshinsky-Moriya interaction corresponding to the scenario described in Sec. IVB left hand side $x-z$ plane, right hand side (circle) $x-y$ plane. The Dzyaloshinsky-Moriya vector $\mathcal{D}_{\mathrm{DM}}$ is oriented perpendicular to the easy axis $K_{e}$ and hard axis anisotropy $K_{h}$. The Dzyaloshinsky-Moriya interaction shows no influence on the profile in this case. Due to the hard axis anisotropy $K_{h}$ we can assume a direct reversal $\mathrm{d} \phi / \mathrm{d} t=0$.

Then, the stability is characterized by:

$$
\phi=\frac{1}{2} \arcsin \left(\frac{M_{S} B_{z}+(\beta-\alpha) \frac{u_{z} M_{S}}{\gamma \Delta}}{\alpha K_{h}}\right) .
$$

and the velocity of the domain wall described by:

$$
v=\frac{\gamma B_{z}}{\alpha} \Delta+\frac{\beta}{\alpha} u_{z} .
$$

\section{Precessional motion}

In this subsection we assume $K_{h}=0$, which means no hard axis anisotropy and therefore a precessional motion $\mathrm{d} \phi / \mathrm{d} t \neq 0$. The Dzyaloshinsky-Moriya vector in this scenario is oriented in $+y$ direction (see Fig. 6). Furthermore, for simplicity we assume that $\mathrm{d} \phi / \mathrm{d} z=0$.

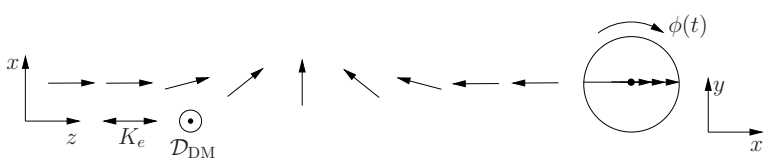

FIG. 6: Sketch of the domain wall profile of a 1D transverse domain wall with Dzyaloshinsky-Moriya interaction corresponding to the scenario described in Sec. IVC left hand side $x-z$ plane, right hand side (circle) $x-y$ plane. The Dzyaloshinsky-Moriya vector $\mathcal{D}_{\mathrm{DM}}$ is oriented perpendicular to the easy axis anisotropy $K_{e}$. Due to the absence of the hard axis anisotropy $K_{h}$ we can assume a precessional motion $\mathrm{d} \phi / \mathrm{d} t \neq 0$ which leads to a time dependent domain wall width $\Delta(t)$ and domain wall energy $E(t)$.

This is definitively the case when the transversal component of the domain wall and the Dzyaloshinsky-Moriya vector $\vec{D}_{\mathrm{DM}}$ are perpendicular as in Fig. 6. In this case the Dzyaloshinsky-Moriya interaction assists the magnetization reversal in the $x-z$ plane which is described by the angle $\theta$. The assumption $\mathrm{d} \phi / \mathrm{d} z=0$ is not necessarily the case when $\vec{D}_{\mathrm{DM}}$ is parallel to the transversal component of the domain wall.
Under these assumptions, especially $\mathrm{d} \phi / \mathrm{d} z=0$, we find:

$$
\frac{\delta \mathcal{E}}{\delta \theta}=2 A \frac{\mathrm{d}^{2} \theta}{\mathrm{d} z^{2}}+2 K_{e} \sin \theta \cos \theta=0
$$

and therefore, the well know domain wall profile (3) together with the domain wall width:

$$
\Delta=\sqrt{\frac{A}{K_{e}}},
$$

and the domain wall energy:

$$
E=4 \sqrt{A K_{e}}+\pi \mathcal{D}_{\mathrm{DM}} .
$$

The variation $\delta \mathcal{E} / \delta \phi$ under the assumption to be in the center of the domain wall $(\theta=\pi / 2)$ leads to:

$$
\left.\frac{\delta \mathcal{E}}{\delta \phi}\right|_{\theta=\pi / 2}=\frac{\mathcal{D}_{\mathrm{DM}}}{\Delta} \sin \phi .
$$

Then, it is easy to write the equations (11) and (12) as:

$$
\frac{v}{\Delta}=\alpha \frac{\mathrm{d} \phi}{\mathrm{d} t}-\frac{\gamma}{M_{S}} \frac{\mathcal{D}_{\mathrm{DM}}}{\Delta} \sin \phi+\frac{u_{z}}{\Delta},
$$

and

$$
\frac{\mathrm{d} \phi}{\mathrm{d} t}=\frac{\alpha v}{\Delta}-\gamma B_{z}-\frac{\beta u_{z}}{\Delta} .
$$

Eliminating $\mathrm{d} \phi / \mathrm{d} t$ leads to the following velocity equation:

$$
v(t)=\frac{\gamma B_{z}}{\alpha+\frac{1}{\alpha}} \Delta+\frac{1+\alpha \beta}{1+\alpha^{2}} u_{z}-\frac{\gamma \mathcal{D}_{\mathrm{DM}} \sin \phi(t)}{M_{S} \Delta\left(1+\alpha^{2}\right)} .
$$

In time average (mean value) the velocity of the domain wall is equal to:

$$
\bar{v}=\frac{\gamma B_{z}}{\alpha+\frac{1}{\alpha}} \Delta+\frac{1+\alpha \beta}{1+\alpha^{2}} u_{z},
$$

which is equal to the velocity of a transverse domain wall with precession and without Dzyaloshinsky-Moriya interaction [11].

Eliminating $v$ in Eq. (46) and (47) lead to the following differential equation for $\phi$ :

$$
\frac{\mathrm{d} \phi}{\mathrm{d} t}=\frac{\alpha-\beta}{1+\alpha^{2}} \frac{u_{z}}{\Delta}-\frac{\gamma B_{z}}{1+\alpha^{2}}-\frac{\alpha \gamma \mathcal{D}_{\mathrm{DM}}}{M_{S} \Delta\left(1+\alpha^{2}\right)} \sin \phi(t) .
$$

This differential equation is needed to be solved to calculate the velocity $v(t)$. For simplification we write the differential equation Eq. (50) as:

$$
\frac{\mathrm{d} \phi}{\mathrm{d} t}=\phi_{0}-\phi_{1} \sin \phi(t)
$$

with

$$
\phi_{0}=\frac{\alpha-\beta}{1+\alpha^{2}} \frac{u_{z}}{\Delta}-\frac{\gamma B_{z}}{1+\alpha^{2}},
$$




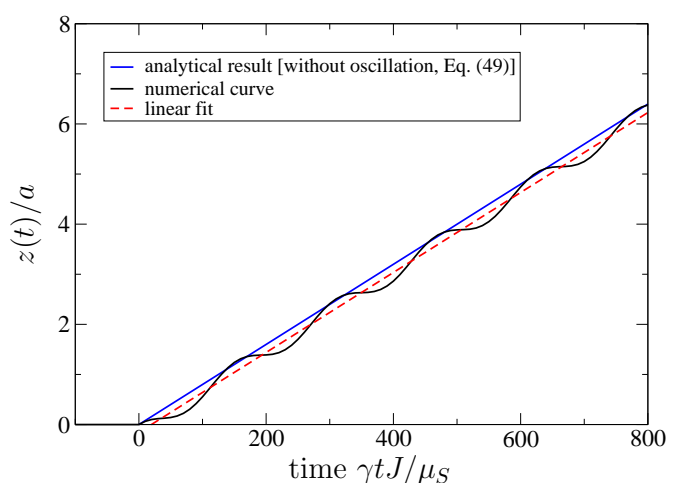

FIG. 7: Domain wall motion of a transverse domain wall with Dzyaloshinsky-Moriya interaction corresponding to the scenario described in Sec. IVC domain wall position as function of time corresponding to the scenario that the DzyaloshinskyMoriya vector $\mathcal{D}_{\mathrm{DM}}$ shows an orientation perpendicular to the easy axis anisotropy $K_{e}$ and the assumption that there is no additional hard axis anisotropy $K_{h}=0$. The last assumption leads to a precessional motion which leads to the oscillation in $z(t)$. The curves coming from computer simulations with: $\mu_{S} B_{z} / J=0.2, D_{\mathrm{DM}} / J=0.004$, and $D_{e} / J=0.005$.

and

$$
\phi_{1}=\frac{\alpha \gamma \mathcal{D}_{\mathrm{DM}}}{M_{S} \Delta\left(1+\alpha^{2}\right)} .
$$

Eq. (51) can be easily solved by separation of variables. The result is:

$$
\phi(t)=2 \arctan \left[\frac{\sqrt{\phi_{0}^{2}-\phi_{1}^{2}}}{\phi_{1}} \tan \left(\frac{\sqrt{\phi_{0}^{2}-\phi_{1}^{2}} t}{2}\right)-\frac{\phi_{0}}{\phi_{1}}\right] .
$$

An interesting situation appears if the domain wall is driven by an electrical current $\left(u_{z} \neq 0, B_{z}=0\right)$ and if we assume $\alpha=\beta$. It is known that in this case a transverse domain wall without Dzyaloshinsky-Moriya interaction shows no precession $\mathrm{d} \phi / \mathrm{d} t=0$ even if the domain wall normally precess $\mathrm{d} \phi / \mathrm{d} t \neq 0$ if $\alpha \neq \beta$. However, in the case with Dzyaloshinsky-Moriya interaction we find a precessional motion even in this special situation: $\alpha=\beta$, $B_{z}=0$. In this case we find:

$$
\phi(t)=2 \arctan \left[\exp \left(-\frac{\alpha \gamma \mathcal{D}_{\mathrm{DM}}}{M_{S} \Delta\left(1+\alpha^{2}\right)} t\right)\right],
$$

and

$$
\bar{v}=u_{z} .
$$

To get a better feeling about the accuracy and correctness of the results of the last subsection additional numerical simulations have been performed. Therefore the Gilbert equation (8) has been solved using the discrete Heisenberg model (11) and as numerical solver the Heun method. Starting point of the simulation was a relaxed domain wall in the first half of a linear chain of the length of 1000 magnetic moments. The orientation of the chain is along the $z$ axis and the magnetic momets inside the domains are in $\pm z$ direction. The unrelaxed domain wall is a $180^{\circ}$ head-to-head transverse domain wall which becomes the final shape during the relaxation process. After switching on an electric current the domain wall displacement has been determined by looking for the zero-crossing of the tanh-profile of the domain wall: $S_{z}=0$. For more details of the computer simulations on the atomic length scale please see 9 -11.

Fig. [7]shows the domain wall displacement as function of time for a current driven transverse domain wall described as in the last scenario with $\alpha<\beta$. As predicted by analytical calculations the domain wall shows an oscillatory behavior and moves with the averaged velocity given by Eq. (49). However due to the oscillation the domain wall arrives the end of the system with a delay or earlier depending on the sign of the DzyaloshinskyMoriya vector $\mathcal{D}_{\mathrm{DM}}$.

\section{SUMMARY}

In summary this manuscript gives a complete overview of the domain wall motion of transverse domain walls under the influence of the Dzyaloshinsky-Moriya interaction. The description is analytical using the $q-\phi$ model and covers the scenarios of a Dzyaloshinsky-Moriya vector parallel respectively perpendicular to the easy axis direction and the fact that the domain wall can show a direct reversal or precessional motion. The first two scenarios describe the situation of a Dzyaloshinsky-Moriya vector parallel to the easy axis direction. These scenarios have been described by Tretiakov and Abanov [6] however using too complex description. The given description within this paper is simpler and therefore easier to understand. The third scenario is identical to the description of Thiaville et al. [7] of a Dzyaloshinsky-Moriya vector perpendicular to easy axis and parallel to the hard axis direction. However, here we give some more informations. The fourth scenario describes the situation of a Dzyaloshinsky-Moriya vector perpendicular to the easy axis and at the same time hard axis anisotropy. In this case the Dzyaloshinsky-Moriya interaction has no influence as long we assume that $\mathcal{D}_{\text {DM }}$ is small with respect to the anisotropies $K_{e}$ and $K_{h}$. The fifth scenario assumes a Dzyaloshinsky-Moriya vector perpendicular to easy axis direction. However, within this scenario we assume a precessional motion. This scenario has not been described so far and compliments the description of the dynamics of transverse domain walls under the influence of a Dzyaloshinsky-Moriya interaction. We have seen that the Dzyaloshinsky-Moriya interaction can lead to a modification of the velocity, or at least the stability condition. Other scenarios show no influence of the DzyaloshinskyMoriya interaction on the velocity. To compliment these results additional computer simulations could be helpful, especially in cases where the domain wall cannot be 
described by a simple transverse domain wall.

\section{Acknowledgments}

This work has been supported by the Deutsche Forschungsgemeinschaft in the framework of subproject
B3 of the SFB 668 and by the Cluster of Excellence "Nanospintronics".
[1] S. S. P. Parkin, M. Hayashi, and L. Thomas, Science 320, 190 (2008).

[2] M. Hayashi, L. Thomas, R. Moriya, C. Rettner, and S. S. P. Parkin, Science 320, 209 (2008).

[3] M. Menzel, Y. Mokrousov, R. Wieser, J. E. Bickel, E. Vedmedenko, S. Blügel, S. Heinze, K. von Bergmann, A. Kubetzka, and R. Wiesendanger, Phys. Rev. Lett. 108, 197204 (2012).

[4] D. A. Allwood, G. Xiong, C. C. Faulkner, D. Atkinson, D. Petit, and R. P. Cowburn, Science 309, 1688 (2005).

[5] A. A. Khajetoorians, J. Wiebe, B. Chilian, and R. Wiesendanger, Science 332, 1062 (2011).

[6] O. A. Tretiakov and A. Abanov, Phys. Rev. Lett. 105, 157201 (2010).

[7] A. Thiaville, S. Rohart, E. Jue, V. Cros, and A. Fert, Europhys. Lett. 100, 57002 (2012).

[8] E. Y. Vedmedenko, L. Udvardi, P. Weinberger, and R. Wiesendanger, Phys. Rev. B 75, 104431 (2007).
[9] R. Wieser, U. Nowak, and K. D. Usadel, Phys. Rev. B 69, 064401 (2004).

[10] C. Schieback, M. Kläui, U. Nowak, U. Rüdiger, and P. Nielaba, Eur. Phys. J. B 59, 429 (2007).

[11] R. Wieser, E. Y. Vedmedenko, P. Weinberger, and R. Wiesendanger, Phys. Rev. B 82, 144430 (2010).

[12] W. F. Brown, Micromagnetics (Wiley, New York, 1963).

[13] A. P. Malozemoff and J. C. Slonczewski, Magnetic Domain Walls in Bubble Materials (Academic Press, New York, 1979).

[14] A. Hubert and R. Schäfer, Magnetic Domains (SpringerVerlag, Berlin, 1998).

[15] T. L. Gilbert, IEEE Trans. Mag. 40, 3443 (2004).

[16] J. C. Slonczewski, J. Magn. Magn. Mat. 159, L1 (1996).

[17] S. Zhang and Z. Li, Phys. Rev. Lett. 93, 127204 (2004).

[18] A. Mougin, M. Cormier, J. P. Adam, P. J. Metaxas, and J. Ferrè, Europhys. Lett. 78, 57007 (2007). 